\title{
Bericht aus dem Arbeitskreis Forensik
}

Eine Fülle von Themen und Problemlagen beschäftigen uns auch weiterhin im Bereich des Maßregelvollzugs.

\section{Unterbringung gemäß §63 StGB}

Im Bereich der Unterbringung gemäß § 63 StGB, betreffend psychisch kranke Straftäter, kommt es nahezu bundesweit zu einer Zunahme von Unterbringungen gemäß §126a StPO. Es handelt sich in der Regel um Patienten, die schon zuvor zum psychiatrischen System Kontakt hatten, an einer schizophrenen Erkrankung leiden und in den meisten Fällen die Behandlung abgebrochen und Drogen konsumiert haben.

Eine Evaluation dieses Phänomens ist bisher nicht erfolgt, wäre aber sicherlich mittelfristig sinnvoll. Damit setzt sich der Trend einer verhältnismäßigen Zunahme psychosekranker Patienten im Maßregelvollzug fort.

Die Folge der Gesetzesänderung mit Erhöhung der prognostischen Gutachtenfrequenzen und der Entlassungen im Rahmen der Verhältnismäßigkeit begleitet uns durchgehend im praktischen Alltag. Wie vermutet und befürchtet verschiebt sich ab 6 Jahren Unterbringung der Schwerpunkt in Richtung des Wartens auf das nächste Gutachten, Verschieben von wichtigen Entscheidungen, insbesondere auch Lockerungsentscheidungen, und Verlust des Fokus auf die Behandlung.

Die vermehrten Entlassungen im Rahmen der Verhältnismäßigkeit fordern auch weiterhin höchsten Einsatz der damit befassten forensischen Nachsorgeambulanzen, resultierend aus der Schwierigkeit, dass Patienten ohne Weisungen und ausschließlich aufgrund juristischer Begründungen entlassen werden.
Ein nicht zu unterschätzender neuer Trend sind Schadensersatzforderungen gegenüber Gutachtern, dies auch in Fällen, in denen ein Gericht bereits ein Urteil auf der Grundlage des Gutachtens gefällt hat und dieses Gutachten damit als fachlich fundiert anerkannt hat. Auch hier wird zu beobachten sein, ob es eine Entwicklung ist, die sich fortsetzt und die natürlich die Motivation zur Erstellung schwieriger Gutachten für junge Mitarbeitende nicht gerade fördern wird.

Zu verweisen wäre noch auf die überarbeiteten Richtlinien zu den Mindestanforderungen für Prognosegutachten, aktuell veröffentlicht u. a. in der NSZT.

\section{Unterbringung gemäß §64 StGB, suchtkranke Straftäter}

Die Probleme im Bereich der Unterbringung suchtkranker Straftäter gemäß §64 StGB haben sich nicht verändert. Weiterhin gibt es auch hier steigende Unterbringungszahlen und viele Ideen und Wünsche nach einer gesetzlichen Regelung, die insbesondere die Frage der Sicherstellung einer Diagnose und einer Festlegung der Mindestbehandlungsdauer betrifft. Zwischenzeitlich hat aber wohl die Konferenz der Justizminister der Bundesländer das Thema aufgegriffen und es bleibt abzuwarten, wie schnell hier eine Dynamik entstehen kann.

\section{$\S 20 / 21$ StGB}

Es gibt aber auch erfreuliche Entwicklungen. So soll der Gesetzestext für den §20/ 21 StGB geändert werden und die Begriffe „Schwachsinn“ und „schwere andere seelische Abartigkeit“ durch die Begriffe „Intelligenzminderung“ und „schwere andere seelische Störung“ ersetzt werden.

\section{Zwangsmaßnahmen}

Zwischenzeitlich gibt es in allen Bundesländern Regelungen für die Durchführung einer medikamentösen Zwangsbehandlung im Maßregelvollzug. Allerdings wird seitens der Genehmigungsstellen, die von Land zu Land variieren, oft eine Medikation vorgegeben, die innerhalb des ohnehin schon sehr bürokratischen Antragsprozesses dann noch zu zusätzlichen Limitierungen führt und teils fragwürdige Vorgaben macht.

Des Weiteren ist auch die richterliche Genehmigung von Fixierungen, die länger als eine halbe Stunde dauern, mittlerweile an die jeweils zuständigen Amtsgerichte delegiert worden.

Diese neuen Regelungen haben insofern Relevanz für den Maßregelvollzug, als nicht nur der Anteil der psychosekranken Patienten zugenommen hat, sondern eben immer mehr nicht behandlungswillige Patienten darunter sind, die bereits zuvor im allgemeinpsychiatrischen System gescheitert sind.

\section{Stichtagserhebungen}

Regelmäßig werden im Arbeitskreis Forensik die Ergebnisse der Stichtagserhebungen zur Unterbringung gemäß §64 StGB vorgestellt. Hier gibt es einen Überblick u.a. über Delikte, Diagnosen, Geschlechtsverteilung, Lockerungsstufen und Verweildauern. Erfreulicherweise wird in Zukunft auch die Stichtagserhebung zur Unterbringung gemäß $§ 63$ StGB wiederaufgenommen werden, sodass es in Ergänzung zu bereits vorhandenen Datensammlungen auch hier relevante ergänzende Ergebnisse geben wird.

\section{Korrespondenzadresse}

Jutta Muysers LVR-Klinik Langenfeld Kölner Straße 82 40764 Langenfeld Jutta.Muysers@lvr.de 